\title{
Melting and Crystallization of Solution Crystallized Ultra-High Molecular Weight Polyethylene under High Pressure
}

\author{
Munehisa Yasuniwa, Masashi Yamaguchi, ${ }^{*}$ Atsushi Nakamura, \\ and Shinsuke TsUBAKIHARA \\ Department of Applied Physics, Faculty of Science, Fukuoka University, \\ Jonan-ku, Fukuoka 814-01, Japan
}

(Received July 10, 1989)

\begin{abstract}
Differential thermal analysis of a ultra-high molecular weight polyethylene (UHMW-PE, $M_{v} ; 2.7 \times 10^{6}$ ) crystallized from a $0.1 \mathrm{wt} \%$ decalin solution was performed at pressures from about atmospheric pressure to $600 \mathrm{MPa}$. In the high pressure region such as $600 \mathrm{PMa}$, thick lamellae similar to extended-chain crystal are formed very rapidly in the heating process. The rapid lamellar thicknening is suggested to be due to the decrease of enthanglements. Its phase diagram was determined. The triple point in the phase diagram was about $370 \mathrm{MPa}$. The high pressure phase region of the solution crystallized sample is between those of as-polymerized powder sample and bulk sample which is melt-crystallized one after kneading in the melt. The change of phase diagram among those samples may be due to differences in entanglement density.

KEY WORDS Polyethylene / Ultra High Molecular Weight / Pressure / Melting / Crystallization / Phase Diagram / Entanglement / Extended-Chain Crystal / Differential Thermal Analysis /
\end{abstract}

In the past ten years, the production of high-strength/high modulus fiber by ultra drawing of high or ultra-high molecular weight polyethylene (UHMW-PE) has been studied extensively. Smith et al. ${ }^{1}$ and Lemstra et al. ${ }^{2}$ suggested that the strongly enhanced drawability is due to reduction of the entanglement density, and that the reduction is achieved by the crystallization from dilute solution.

Melting and crystallization processes of PE under high pressure have been investigated by many authors. However, investigation was focused on bulk samples of low to high molecular weight PE. Recently, Yasuniwa et $a l .{ }^{5-7}$ reported melting and crystallization processes of as-polymerized powder and bulk samples of UEMW-PE under high pressure. There are obviously certain differences of the melting and crystallization behavior between these two types of samples. They suggested that entanglements between PE-chains have an important effect on the melting and crystallization behavior under high pressure. Accordingly, the melting and crystallization processes under high pressure of solution-crystallized UHMW-PE, in which the enthanglements are reduced effectively, are problems to be solved.

In the present investigation, the melting and crystallization processes under high pressure of a solution-crystallized UHMW-PE are studied, and its phase diagram was determined. In addition, the effects of entanglements between PE-chains on the formation of the high pressure phase are discussed.

\section{EXPERIMENTAL}

Commercial grade unfractionated high den-

* Present address: Kyushu Matsushita Electric Co., Ltd., Minoshima 4-1-62, Hakata-ku, Fukuoka 812, Japan. 
sity UHMW-PE, as-polymerized powder sample (Mitsui Petrochemical Industries Co.; Hizex Million 340M), was used in this work. The viscosity averaged molecular weight $\left(M_{v}\right)$ of the sample measured by supplier is $2.7 \times$ $10^{6}$.

A decalin solution of $0.1 \mathrm{wt} \%$ of the powder sample with $2 \mathrm{wt} \%$ polymer of 2,6-di- $t$-butyl$p$-cresol as antioxidant was prepared at $160^{\circ} \mathrm{C}$ for $40 \mathrm{~min}$ under nitrogen. The solution was cooled to room temperature in an oil bath at the cooling rate of about $0.2 \mathrm{~K} \mathrm{~min}^{-1}$. The sedimented polymer was collected with a glass filter, then the polymer was dried in a vacuum oven for several hours after evaporation of the solvent in an atmosphere for two weeks. The small angle X-ray scattering profiles for the solution crystallized sample (hereafter referred to as $S$ ) showed a broad but distinct peak at $2 \theta=0.7^{\circ}$. Since $\mathrm{Cu}-K_{\alpha}$ radiation was used as an incident $\mathrm{X}$-ray beam, the angle of $0.7^{\circ}$ corresponds approximately to the long period of $12 \mathrm{~nm}$. That is, $S$ consists of folded-chain crystal (FCC) whose lamellar thickness is approximately $10 \mathrm{~nm}$ with wide distribution.

Matsuo et al. ${ }^{3,4}$ measured the relationship between reduced viscosity and concentration of decalin solution of UHMW-PE $\left(M_{v} ; 6 \times 10^{6}\right)$ to get optimum conditions for drawing of dry gel film. The reduced viscosity showed a weak concentration dependence in the regime of low concentration (lower than $0.1 \mathrm{wt} \%$ ). In contrast, it showed strong concentration dependence in the regime of high concentration (higher than $0.44 \mathrm{wt} \%$ ). They suggested that most of the chain molecules in the regime of low concentration are random coils having coupling entanglements that will be predominantly intra-molecular in nature. On the other hand, they thought that solutions corresponding to the regime of high concentration consist of interpenetrating random coils which form a large number of coupling entanglements that are both intra- and intermolecular. In the present experiment, molecular weight of $S$ is lower than their sample, and $S$ was made from $0.1 \mathrm{wt} \%$ solution. Therefore, it is evident from their data that entanglement density of $S$ is considerably low level, and that entanglement between inter-molecular chain is very small.

Differential thermal analysis (DTA) under high pressure was carried out by the use of an apparatus used in a series of our works. ${ }^{5-7}$ DTA curves were obtained in the following process designated as run 1 -run 3 successively. Run 1 is the melting process of $S$ (heating rate: $5 \mathrm{~K} \mathrm{~min}^{-1}$. Run 2 is the crystallization process by slow cooling (cooling rate; $2 \mathrm{~K} \mathrm{~min}^{-1}$ ). Run 3 is the melting process of the sample crystallized through the process of run 2 (heating rate, $5 \mathrm{~K} \mathrm{~min}^{-1}$ ).

\section{RESULTS AND DISCUSSION}

High pressure DTA of $S$ was carried out up to about $600 \mathrm{MPa}$. Peaks due to the melting (or crystallization) of FCC, extended-chain crystal (ECC) and the high pressure phase in the subsequent DTA curves are denoted by "F", "E", and "H", respectively. Assignments of DTA peaks are based on ref 8 in which melting and crystallization processes of a bulk sample of normal molecular weight $\operatorname{PE~}\left(M_{w}\right.$; $4.2 \times 10^{4}$ ) under high pressure have been studied. Since the high pressure DTA cell used in this work is an inner heating type, applied pressure changes slightly with temperature. DTA curves presented in Figures 1 to 3 are corrected on the basis of the pressure dependence of melting or crystallization temperature.

Figure 1 shows DTA curves obtained at about $300 \mathrm{MPa}$. Run 1 shows the melting process of $S$. As lamellar thickening occurs successively in the heating process, the peak of run 1 does not directly correspond to the melting of the FCC lamellae of the initial thickness. The low and high temperature peaks of run 3 are due to the melting of FCC and ECC, respectively. These peaks in Figure 1 are slightly sharper than corresponding DTA 


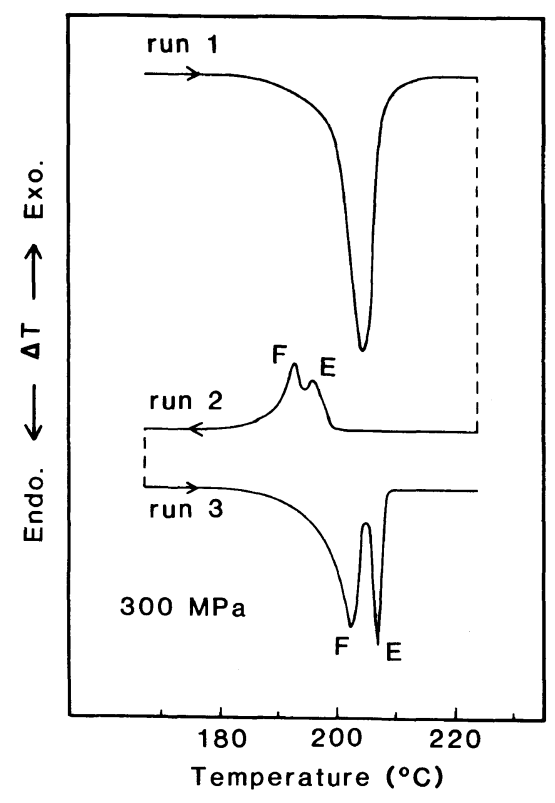

Figure 1. DTA curves of melting and crystallization processes of a solution crystallized sample of UHMW-PE (sample $S$ ) at about 300 PMa.

peaks obtained at $3000 \mathrm{~kg} \mathrm{~cm}^{-2}(290 \mathrm{MPa})$ for normal molecular weight PE. ${ }^{8}$

Figure 2 shows DTA curves obtained at about $585 \mathrm{MPa}$. The high temperature peak of run 1 is due to the melting of the high pressure phase. The high and low temperature peaks of run 2 are due to the crystallization to the high pressure phase and ECC, respectively. Low and high temperature peaks in run 3 are due to the melting of ECC and the high pressure phase, respectively. As shown in the figure, the low temperature peak of run 1 is sharp and not so different from that of run 3 . In addition, there is not a distinct peak below the low temperature peak. These results indicate successive lamellar thickening and rapid formation of very thick lamellae in the heating process of $S$. The thickening of the lamellae may be similar to that of ECC.

Tsubakihara et al. $^{9}$ studied the formation mechanism of ECC under high pressure by the use of single crystal mats of high molecular weight $\operatorname{PE}\left(M_{v} ; 2.2 \times 10^{5}\right)$. They obtained wide

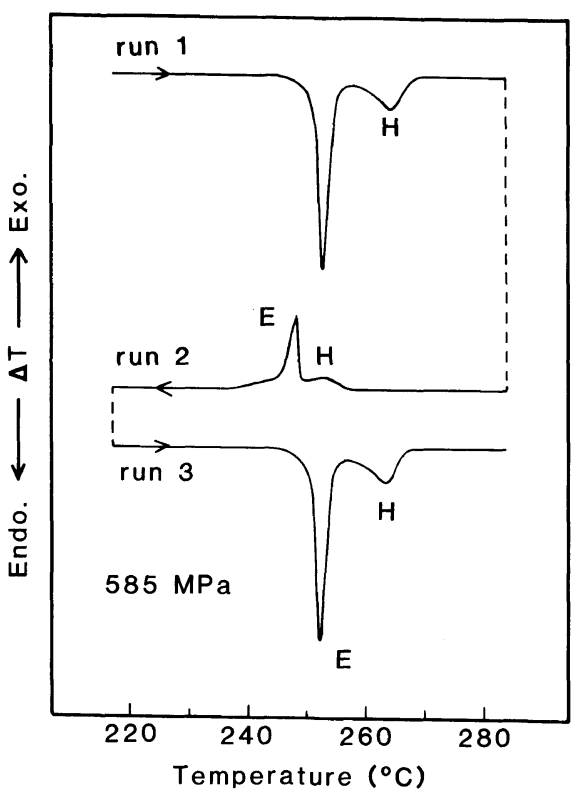

Figure 2. DTA curves of melting and crystallization processes of a solution crystallized sample of UHMW-PE (sample $S$ ) at about $585 \mathrm{MPa}$.

angle X-ray diffraction patterns and small angle X-ray scattering patterns of the single crystal mats annealed under high pressure and have reported the changes of lamellar thickness and orientational direction of $c$-axis of molecular chains in the single crystal mats. They also measured increase of the lamellar thickness in the heating process under high pressure and obtained the results of rapid lamellar thickening at higher pressure (500 $\mathrm{MPa}) .{ }^{10}$ Namely, the present suggestion on the lamellar thickening obtained by the high pressure DTA is confirmed by their results of the X-ray measurements.

High pressure DTA curves of UHMW-PE $\left(M_{v} ; 2.5 \times 10^{6}\right)$ have already been presented for powder and bulk samples by Yasuniwa et al. ${ }^{5}$ The bulk samples were melt-crystallized once after kneading the powder sample in the melt. The detailed procedure for the preparation of the bulk sample is mentioned elsewhere. ${ }^{5,6}$ The DTA curves of the bulk sample show very broad peaks and are considerably different 
from those of $S$. In contrast, the DTA curves of $S$ are close to corresponding to the DTA curves of the powder sample. However, the low temperature peak of run 1 of the powder sample is broader than the present ones. The corresponding DTA peak of normal molecular weight PE is also broad. ${ }^{8}$ These results can be explained as follows by the use of entanglement concept. In the powder sample and the normal molecular weight PE, the entanglement density is very high. Since entanglements hinder chain extension, i.e., lamellar thickening, in the heating process, the low temperature peak of run 1 for these samples is broad. In the case of $S$, the reduction of the entanglement density results in rapid formation of the very thick lamellae and gives a sharp melting peak, as shown in run 1 of Figure 2.

Takamizawa et al. ${ }^{11}$ reported high pressure DTA curves for a solution crystallized PE whose molecular weight is $4.5 \times 10^{4}$. However, their DTA curves are different from present ones. There are extra endo- and exothermic peaks in the low temperature region of their DTA curves obtained above $4 \mathrm{~kb}$ (390 MPa). Differences in the DTA curves may possibly be due to differences in molecular weight, because similar differences in the DSC curves have been observed at atmospheric pressure. ${ }^{12,13}$

Figure 3 shows melting DTA curves of pressure-crystallized sample (run 3) of $S$ at pressures from about 200 to $600 \mathrm{MPa}$. As shown in this figure the peak area of the low temperature peak which corresponds to FCC decreases with increasing pressure. In contrast, the high temperature peak which corresponds to the high pressure phase is separated from the peak of ECC above about $400 \mathrm{MPa}$. The peak area is almost constant even with increasing pressure. These DTA curves are similar to reported ones for normal molecular weight $\mathrm{PE}^{8}$ except for the disappearance of FCC peak in the low temperature region above about $500 \mathrm{MPa}$. The melting temperatures of FCC, ECC, and the high pressure phase are

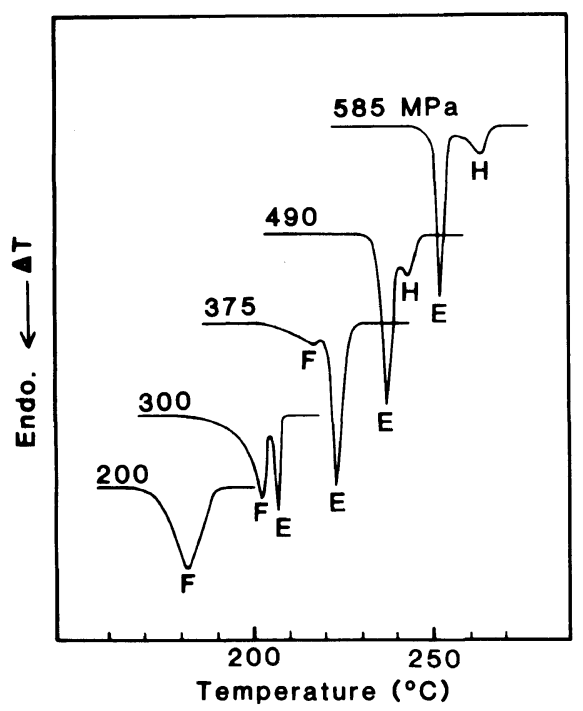

Figure 3. Melting DTA curves of pressure-crystallized samples (run 3) of $S$ at pressures indicated in the figure.

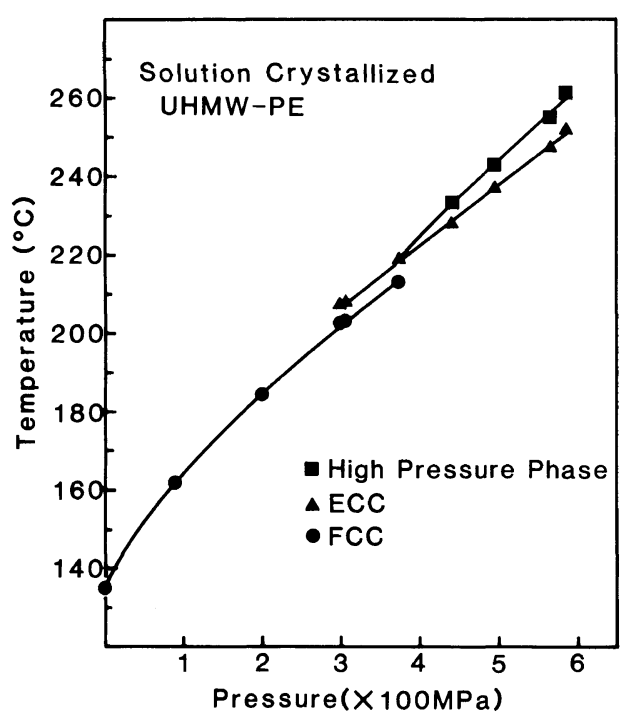

Figure 4. Phase diagram of a pressure-crystallized sample of $S$.

obtained from the peaks of the DTA curves in Figure 3. The phase diagram of $S$ is determined by the plot of the peak temperatures, and is shown in Figure 4. The high pressure phase appears above about $370 \mathrm{MPa}$. That is, the triple point of the phase diagram for melting can be found at about $370 \mathrm{MPa}$. 
It has been shown ${ }^{5}$ that the high pressure phase region for an as-polymerized powder sample of UHMW-PE is higher in pressure and temperature than that of the bulk sample. The present experiment shows that the high pressure phase region of the pressure-crystallized sample of $S$ is between those of these samples. This can be explained as follows. Entanglements hinder the crystal growth, and defects result in the crystal. Therefore crystals become smaller and more disordered with increasing entanglements, resulting in decrease of the melting temperature. In contrast the melting temperature increases by superheating which is also caused by the entanglements. As discussed in the previous paper, ${ }^{5}$ the molecular chain length of UHMW-PE is too long; during crystallization under high pressure the chain disentangling would be difficult to occur on the time scale of the present experimental condition. Therefore the entanglements should be trapped in the sample. Since the former effect is large in the bulk sample by too many entanglements, the bulk sample shows the lowest melting temperature. In contrast, the latter effect is large in the powder sample, therefore the powder sample shows the highest melting temperature. The entanglement density of $S$ is the lowest among these samples, so these two effects are not prominent in the pressurecrystallized sample of $S$. Consequently it shows intermediate high pressure phase region between the powder and bulk sample.
Acknowledgements. The authors should like to thank Professor C. Nakafuku in Kochi University and Mr. R. Campbell in University of Tennessee for a critical reading of the manuscript. This work was supported in part by a Grant-in-Aid for Scientific Research from the Ministry of Education, Science, and Culture of Japan.

\section{REFERENCES}

1. P. Smith, P. J. Lemstra, and H. C. Booij, J. Polym. Sci., Polym. Phys. Ed., 19, 877 (1981).

2. P. J. Lemstra, N. A. J. M. van Aerle, and C. W. M. Bastiaansen, Polym. J., 19, 85 (1987).

3. M. Matsuo, Nihon Reoroji Gakkaishi, 13, 4 (1985).

4. C. Sawatari, T. Okumura, and M. Matsuo, Polym. J., 18, 741 (1986).

5. M. Yasuniwa, K. Haraguchi, C. Nakafuku, and S. Hirakawa, Polym. J., 17, 1209 (1985).

6. M. Yasuniwa and C. Nakafuku, Polym. J., 19, 805 (1987).

7. M. Yasuniwa, S. Tsubakihara, and C. Nakafuku, Polym. J., 20, 1075 (1988).

8. M. Yasuniwa, C. Nakafuku, and T. Takemura, Polym. J., 4, 526 (1973).

9. S. Tsubakihara, M. Yamaguchi, and M. Yasuniwa, Rept. Prog. Polym. Phys. Jpn., 31, 159 (1988).

10. M. Yasuniwa, M. Yamaguchi, and S. Tsubakihara, in preparation.

11. K. Takamizawa, A. Ohno, and Y. Urabe, Polym. J., 7, 342 (1975).

12. L. Mandelkern and A. L. Allou, J. Polym. Sci., C, 4, 447 (1966).

13. B. Wunderlich, "Macromolecular Physics," Vol. 3, Academic Press, New York, N.Y., 1980, Chapter 9. 